PROCEEDINGS OF THE

AMERICAN MATHEMATICAL SOCIETY

Volume 34, Number 2, August 1972

\title{
EXTENDING A COMPLETE CONVEX METRIC
}

\author{
ROBERT A. DOOLEY ${ }^{1}$
}

\begin{abstract}
A metric $D$ is convex if for every two points $x, z$ there is a third point $y$ such that $D(x, y)+D(y, z)=D(x, z)$. A generalized continuum is a connected, locally compact, separable metric space. It is shown that if $M_{1}$ is a space with a complete convex metric $D_{1}$ and $M_{2}$ is a locally connected generalized continuum whose intersection with $M_{1}$ is nonempty and compact, there is a complete convex metric for $M_{1} \cup M_{2}$ that extends $D_{1}$. Using this result, four classes of locally connected generalized continua are characterized by the type of complete convex metric they admit.
\end{abstract}

1. Introduction. A metric $D$ is said to be convex if for every two points $x, z$ there is a third point $y$, called a between point of $x$ and $z$, such that $D(x, y)+D(y, z)=D(x, z)$. If in addition $D(x, y)=D(y, z)=$ $D(x, z) / 2$, the point $y$ is called a midpoint of $x$ and $z$. A linear set in a metric space is one that is isometric with a subset of the real line; a linear arc is called a segment. A generalized continuum is a connected, locally compact, separable metric space. A Peano continuum is a connected, locally connected, compact metric space.

In 1949 Bing [3] and Moise [9] independently solved a long-standing problem by showing that every Peano continuum admits a convex metric. Further, Bing has shown [2] that if $M_{1}$ and $M_{2}$ are intersecting Peano continua and $D_{1}$ is a convex metric for $M_{1}$, there is a convex metric $D_{3}$ for $M_{1} \cup M_{2}$ that extends $D_{1}$. It is understood that the topology induced by $D_{3}$ on $M_{1} \cup M_{2}$ preserves the given topology of $M_{2}$.

In 1955 Tominaga and Tanaka [10] showed, by a method similar to that which Bing used to prove the convexifiability of Peano continua, that every locally connected generalized continuum admits a complete convex metric. The present paper addresses the question: Under what

Presented to the Society, August 20, 1971; received by the editors August 13, 1971. AMS 1970 subject classifications. Primary 54E50; Secondary 54F15.

Key words and phrases. Convex metric, generalized continuum, strongly convex, without ramifications, simple triod, interval, simple closed curve.

${ }^{1}$ This research was supported by NSF Grant GZ-1694 and comprises a part of the author's dissertation at Oklahoma State University under the direction of Professor John Jobe.

(c) American Mathematical Society 1972 
conditions can a complete convex metric be extended to a locally connected generalized continuum? By defining the metric $D_{3}$ of Bing [2] in a more general setting and by noting a special property of this metric, the following result is established: If $M_{1}$ is a space with a complete convex metric $D_{1}$ and $M_{2}$ is a locally connected generalized continuum whose intersection with $M_{1}$ is nonempty and compact, there is a complete convex metric $D_{3}$ for $M_{1} \cup M_{2}$ that extends $D_{1}$. In this case, the topology induced by $D_{3}$ is the largest one on $M_{1} \cup M_{2}$ that preserves the given topology of each $M_{i}$.

2. Defining the metric. Let $M_{1}$ and $M_{2}$ be intersecting Peano continua and let $D_{i}$ be a convex metric for $M_{i}$. In [2], the metric $D_{3}$ was defined in the following way. Let $F(x), x>0$, be a real-valued function which is never less than

$$
\sup \left\{D_{1}(p, q): p, q \in M_{1} \cap M_{2}, D_{2}(p, q) \leqq x\right\}
$$

and such that $F(x)$ approaches zero as $x$ approaches zero, $F^{\prime}(x)$ is a continuous monotone nonincreasing function with $F^{\prime}(x)>1$, and the improper integral

$$
\int_{0}^{d} F^{\prime}(x) d x
$$

exists for each $d>0$. For every two points $x, y$ of $M_{2}$ let $A(x, y)$ be the set of all $D_{2}$ rectifiable arcs $C$ from $x$ to $y$ that lie, except for possibly their endpoints, in $M_{2} \backslash M_{1}$ and for which the (possibly improper) Riemann integral

$$
\int_{C} F^{\prime}\left[D_{2}\left(p(s), M_{1}\right)\right] d s
$$

exists. Here $s$ denotes $D_{2}$ length along $C$ from a fixed endpoint and $p(s)$ is the point of $C$ whose $D_{2}$ distance along $C$ from the fixed endpoint is $s$. If $x$ lies in $M_{2} \backslash M_{1}, y$ is a point of $M_{1}$ such that $D_{2}(x, y)=D_{2}\left(x, M_{1}\right)$, and $[x y]$ is a $D_{2}$ segment from $x$ to $y$, then

$$
\int_{[x y]} F^{\prime}\left[D_{2}\left(p(s), M_{1}\right)\right] d s
$$

exists and has the value $F\left[D_{2}\left(x, M_{1}\right)\right]$. For all points $x, y$ of $M_{2}$ with $A(x, y) \neq \varnothing$, let

$$
D_{0}(x, y)=\inf \left\{\int_{C} F^{\prime}\left[D_{2}\left(p(s), M_{1}\right)\right] d s: C \in A(x, y)\right\} .
$$

If $D_{0}(x, y)$ exists for two points $x$ and $y$ then $D_{0}(x, y) \geqq D_{2}(x, y)$, and $D_{0}(x, y) \geqq D_{1}(x, y)$ if $x$ and $y$ are points of $M_{1}$. Define $D_{3}$ on $M_{1} \cup M_{2}$ as 
follows: if $x, y \in M_{1}$, then $D_{3}(x, y)=D_{1}(x, y)$; if $x \in M_{1}$ and $y \in M_{2} \backslash M_{1}$, then $D_{3}(x, y)=D_{3}(y, x)=\inf \left\{D_{1}(x, a)+D_{0}(a, y): a \in M_{1}, A(a, y) \neq \varnothing\right\}$; if $x, y \in M_{2} \mid M_{1}$, then $D_{3}(x, y)$ is the minimum of $D_{0}(x, y)$ and

$\inf \left\{D_{0}(x, a)+D_{1}(a, b)+D_{0}(b, y): a, b \in M_{1}, A(x, a) \neq \varnothing, A(b, y) \neq \varnothing\right\} ;$

if $x=y$, then $D_{3}(x, y)=0$. It follows that $D_{3}$ is a metric on $M_{1} \cup M_{2}$, whose restriction to $M_{2}$ is equivalent to $D_{2}$. The topology induced on $M_{1} \cup M_{2}$ by $D_{3}$ is such that $M_{1} \backslash M_{2}$ and $M_{2} \backslash M_{1}$ are separated sets.

3. Properties of the metric. For the definitions and properties of $\S 2$ to hold, it is not necessary that $M_{1}$ and $M_{2}$ be compact. It suffices that $M_{1} \cup M_{2}$ be nonempty and compact and that every two points of $M_{2}$ lie on a $D_{2}$ segment. Menger [8] has shown that such segments exist if $D_{2}$ is complete and convex. For Lemmas $1-4$, let $M_{1}$ be any space with a complete convex metric $D_{1}$, let $M_{2}$ be a locally connected generalized continuum with a complete convex metric $D_{2}$ such that $M_{1} \cup M_{2}$ is nonempty and compact, and let $D_{0}$ and $D_{3}$ be as defined in $\S 2$.

LeMma 1. If $x$ is in $M_{2} \backslash M_{1}$ and $y$ is a point of $M_{1}$ such that $D_{2}(x, y)=$ $D_{2}\left(x, M_{1}\right)$, then $D_{0}(x, y)=\inf \left\{D_{0}(x, a): a \in M_{1}, A(x, a) \neq \varnothing\right\}$.

Proof. With $x$ and $y$ as given, it suffices to show that for any point $a$ in $M_{1}$ with $A(x, a) \neq \varnothing$ and for any $C \in A(x, a)$, then

$$
\int_{[x y]} F^{\prime}\left[D_{2}\left(q(s), M_{1}\right)\right] d s \leqq \int_{C} F^{\prime}\left[D_{2}\left(p(s), M_{1}\right)\right] d s,
$$

where [xy] is a $D_{2}$ segment from $x$ to $y, q(s)$ is the point $u$ of $[x y]$ with $D_{2}(y, u)=s$, and $p(s)$ is the point of $C$ whose $D_{2}$ distance along $C$ from $a$ is $s$. In fact, inequality (1) shows that

$$
D_{0}(x, y)=\int_{[x y]} F^{\prime}\left[D_{2}\left(q(s), M_{1}\right)\right] d s,
$$

obtained by setting $a=y$.

Let $\alpha$ and $\beta$ be the $D_{2}$ lengths of $[x y]$ and $C$, respectively. Since

$$
D_{2}\left(p(s), M_{1}\right) \leqq D_{2}(p(s), a) \leqq s
$$

and $F^{\prime}$ is monotone nonincreasing, then

$$
F^{\prime}\left[D_{2}\left(q(s), M_{1}\right)\right]=F^{\prime}(s) \leqq F^{\prime}\left[D_{2}\left(p(s), M_{1}\right)\right] \text { for } 0<s \leqq \beta .
$$

Further, since $0<\alpha=D_{2}\left(x, M_{1}\right) \leqq \beta$, then

$$
\int_{0}^{\alpha} F^{\prime}\left[D_{2}\left(q(s), M_{1}\right)\right] d s \leqq \int_{0}^{\beta} F^{\prime}\left[D_{2}\left(p(s), M_{1}\right)\right] d s
$$


follows by elementary properties of improper integrals, and is the desired inequality (1).

LEMMA 2. For every point $x$ of $M_{2}, D_{3}\left(x, M_{1}\right) \geqq D_{2}\left(x, M_{1}\right)$.

Proof. Assume $x$ is in $M_{2} \backslash M_{1}$. Let $y$ be a point of $M_{1}$ such that $D_{2}(x, y)=D_{2}\left(x, M_{1}\right)$ and let $z$ be any point of $M_{1}$. Then from Lemma 1 and $\S 2$,

$$
\begin{aligned}
D_{3}(x, z) & =\inf \left\{D_{0}(x, a)+D_{1}(a, z): a \in M_{1}, A(x, a) \neq \varnothing\right\} \\
& \geqq \inf \left\{D_{0}(x, a): a \in M_{1}, A(x, a) \neq \varnothing\right\}=D_{0}(x, y) \\
& \geqq D_{2}(x, y)=D_{2}\left(x, M_{1}\right) .
\end{aligned}
$$

Since $z$ is arbitrary, it follows that $D_{3}\left(x, M_{1}\right) \geqq D_{2}\left(x, M_{1}\right)$.

Lemma 3. Every closed and $D_{3}$ bounded subset of $M_{2}$ is compact.

Proof. By Lemma 2 such a set is also $D_{2}$ bounded, hence is compact according to the generalized Bolzano-Weierstrass theorem proved by Lelek and Mycielski [6] for a locally compact space with a complete convex metric.

Lemma 4. $D_{3}$ is complete and convex.

Proof. The completeness of $D_{3}$ is a result of Lemma 3 and the completeness of $D_{1}$. Convexity is proved by applying the local compactness of $M_{2}$ in much the same way that compactness was used in the result of Bing [2].

THEOREM 1. If $M_{1}$ is a space with a complete convex metric $D_{1}$ and $M_{2}$ is a locally connected generalized continuum whose intersection with $M_{1}$ is nonempty and compact, there is a complete convex metric for $M_{1} \cup M_{2}$ that extends $D_{1}$.

Proof. Tominaga and Tanaka [10] have shown that $M_{2}$ has a complete convex metric $D_{2}$. If $D_{3}$ is constructed from $D_{1}$ and $D_{2}$ as in $\S 2$, then $D_{3}$ extends $D_{1}$, is equivalent to $D_{2}$ on $M_{2}$, and is complete and convex by Lemma 4 .

It should be noted that the topology induced on $M_{1} \cup M_{2}$ by the metric $D_{3}$ given above is the largest topology that preserves the original topologies of the spaces $M_{i}$, since $M_{1} \backslash M_{2}$ and $M_{2} \backslash M_{1}$ are separated sets in this topology.

4. Characterizing locally connected generalized continua by their complete convex metrics. By use of Theorem 1 certain classes of locally connected generalized continua can be identified by metric properties. 
The following two definitions are equivalent to those discussed by Lelek and Nitka [7]. A complete convex metric is said to be strongly convex (SC) if every two points have a unique midpoint. A complete convex metric is without ramifications (WR) if whenever $y$ is a midpoint of $x$ and $z$, and also of $x$ and $z^{\prime}$, then $z=z^{\prime}$. A preliminary proposition is given, whose proof requires only a slight modification of a proof of Blumenthal $[4$, p. 41].

Proposition. If $p$ and $q$ are two points of a complete convex metric space and if $L$ is a linear set consisting of $p, q$, and between points of $p$ and $q$, there is a segment from $p$ to $q$ containing $L$.

THEOREM 2. A locally connected generalized continuum contains no simple closed curve if and only if every complete convex metric for it is strongly convex.

Proof. Suppose the space $M$ contains no simple closed curve. Let $D$ be a complete convex metric for $M$, let $x$ and $z$ be two points of $M$, and suppose $y$ and $y^{\prime}$ are midpoints of $x$ and $z$. By the above proposition there are segments $S$ and $S^{\prime}$ joining $x$ to $z$ and containing $y$ and $y^{\prime}$ respectively. If $y \neq y^{\prime}$, then $S \cup S^{\prime}$ contains a simple closed curve. Thus, $y=y^{\prime}$ and $D$ is $\mathrm{SC}$.

Now suppose that $M$ contains a simple closed curve $C$. A homeomorphism from the unit circle in the plane onto $C$ will induce a complete convex metric $D_{1}$ on $C$ that is not SC-for example, the metric induced on $C$ from the arc length metric on the unit circle. By Theorem 1 there is an extension of $D_{1}$ to a complete convex metric $D_{3}$ for $M$, and $D_{3}$ is not SC.

THEOREM 3. A locally connected generalized continuum contains no simple triod if and only if every complete convex metric for it is without ramifications.

Proof. Let $D$ be a complete convex metric for the space $M$, and suppose $D$ is not WR. Then there are four points $x, y, z, z^{\prime}$ of $M$ such that $y$ is a midpoint of $x$ and $z$, and also of $x$ and $z^{\prime}$. If $[x y],[y z]$, and $\left[y z^{\prime}\right]$ are segments joining $y$ to each of the three other points, then $[x y] \cup[y z] \cup\left[y z^{\prime}\right]$ must contain a simple triod.

Conversely, suppose $M$ contains a simple triod $T$. Since $T$ is homeomorphic with a triod $T^{\prime}$ in the plane composed of three equal line segments meeting at a point, the arclength metric on $T^{\prime}$ will induce a complete convex metric $D_{1}$ on $T$ that is not WR. By Theorem 1 , the metric $D_{1}$ extends to a complete convex metric $D_{3}$ for $M$, and $D_{3}$ is not WR. 
THEOREM 4. A locally connected generalized continuum is homeomorphic to an interval of the real line if and only if every complete convex metric for it is both strongly convex and without ramifications.

Proof. If the space $M$ is homeomorphic to an interval of the real line, then by Theorems 2 and 3 every complete convex metric for $M$ is SC and WR.

On the other hand suppose every complete convex metric for $M$ is both $\mathrm{SC}$ and WR. Let $D$ be a particular complete convex metric for $M$, let $x$ and $z$ be two points of $M$, and let $y$ denote the unique midpoint of $x$ and $z$. Suppose $y^{\prime}$ is a point of $M$ distinct from $y$ such that $D\left(x, y^{\prime}\right)=$ $D\left(y^{\prime}, z\right)$. Let $[x y],[y z]$, and $\left[y y^{\prime}\right]$ be segments from $y$ to $x, z$ and $y^{\prime}$ respectively. If either of the points $x$ or $z$ lies on $\left[y y^{\prime}\right]$ then $[x y] \cup[y z] \cup\left[y y^{\prime}\right]$ would contain a simple closed curve. If neither $x$ nor $z$ lies on $\left[y y^{\prime}\right]$ then $[x y] \cup[y z] \cup\left[y y^{\prime}\right]$ would contain a simple triod. Since by Theorems 2 and 3 both alternatives are impossible, then $y$ itself is the only point of $M$ such that $D(x, y)=D(y, z)$. Since $M$ is a connected metric space in which every two points have exactly one point equidistant from them, then according to Berard [1], $M$ is homeomorphic to an interval of the real line.

THEOREM 5. A locally connected generalized continuum is a simple closed curve if and only if every complete convex metric for it is without ramifications but is not strongly convex.

Proof. If the space $M$ is a simple closed curve, then by Theorem 3 every convex metric for $M$ is WR. However, since $M$ is not contractible to a point, $M$ admits no SC metric [5].

If every complete convex metric on $M$ is $\mathrm{WR}$ but not $\mathrm{SC}$, then by Theorem 2, $M$ must contain a simple closed curve $C$ and, by Theorem 3, every point of $M$ must lie on $C$.

If only compact spaces are considered, Theorems 2 through 5 yield results similar to those obtained by Toranzos [11].

\section{REFERENCES}

1. A. D. Berard, Jr., Characterizations of metric spaces by use of their midsets: intervals, Fund. Math. 73 (1971), 1-7.

2. R. H. Bing, A convex metric for a locally connected continuum, Bull. Amer. Math. Soc. 55 (1949), 812-819. MR 11, 194.

3. - Partitioning a set, Bull. Amer. Math. Soc. 55 (1949), 1101-1110. MR 11, 733.

4. L. M. Blumenthal, Theory and applications of distance geometry, Clarendon Press, Oxford, 1953. MR 14, 1009. 
5. K. Borsuk, On a metrization of polytopes, Fund. Math. 47 (1959), 325-341. MR 22 \#2980.

6. A. Lelek and J. Mycielski, On convex metric spaces. IV, Fund. Math. 61 (1967), 171-176. MR 36 \#4520.

7. A. Lelek and W. Nitka, On convex metric spaces. I, Fund. Math. 49 (1960/61), 183-204. MR 23 \#A2192.

8. K. Menger, Untersuchungen über allgemeine Mètrik, Math. Ann. 100 (1928), 75-163.

9. E. E. Moise, Grille decomposition and convexification theorems for compact metric locally connected continua, Bull. Amer. Math. Soc. 55 (1949), 1111-1121. MR 11, 734.

10. A. Tominaga and T. Tanaka, Convexification of locally connected generalized continua, J. Sci. Hiroshima Univ. Ser A 19 (1955), 301-306. MR 17, 1230.

11. F. Toranzos, Spaces that admit only a certain type of convex metric, Nieuw Arch. Wisk. (3) 14 (1966), 252-254. MR 34 \#5058.

Department of Mathematics and Statistics, OKlahoma State University, Stillwater, OKLahoma 74074 